\title{
Central obesity is predictive of persistent storage lower urinary tract symptoms (LUTS) after surgery for benign prostatic enlargement: results of a multicentre prospective study
}

\author{
Mauro Gacci, Arcangelo Sebastianelli, Matteo Salvi, Cosimo De Nunzio*, Andrea

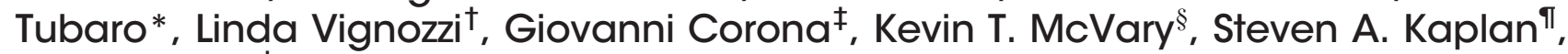 \\ Mario Maggi ${ }^{\dagger}$, Marco Carini and Sergio Serni \\ Department of Urology, Careggi Hospital, University of Florence, Florence, *Department of Urology, Sant'Andrea \\ Hospital, University 'La Sapienza', Rome, 'Department of Clinical Physiopathology, University of Florence, Florence, \\ ${ }^{\ddagger}$ Endocrinology Unit, Medical Department, Maggiore-Bellaria Hospital, Bologna, Italy, ${ }^{\varepsilon}$ Department of Urology, Southern \\ Illinois University School of Medicine, Springfield, IL, and "Department of Urology, Weill Cornell Medical College, Cornell \\ University, New York, NY, USA
}

\section{Objective}

To evaluate the impact of components of metabolic syndrome (MetS) on urinary outcomes after surgery for severe lower urinary tract symptoms (LUTS) due to benign prostatic enlargement (BPE), as central obesity can be associated with the development of BPE and with the worsening of LUTS.

\section{Patients and Methods}

A multicentre prospective study was conducted including 378 consecutive men surgically treated for large BPE with simple open prostatectomy (OP) or transurethral resection of the prostate (TURP), between January 2012 and October 2013. LUTS were measured by the International Prostate Symptom Score (IPSS), immediately before surgery and at 6-12 months postoperatively. MetS was defined according the USA National Cholesterol Education Program-Adult Treatment Panel III.

\section{Results}

The improvement of total and storage IPSS postoperatively was related to diastolic blood pressure and waist circumference (WC). A WC of $>102 \mathrm{~cm}$ was associated with a higher risk of an incomplete recovery of both total IPSS (odds ratio $[\mathrm{OR}] 0.343, P=0.001$ ) and storage IPSS (OR $0.208, P<0.001$ ), as compared with a $\mathrm{WC}$ of $<102 \mathrm{~cm}$. The main limitations were: (i) population selected from a tertiary centre, (ii) Use exclusively of IPSS questionnaire, and (iii) No inclusion of further data.

\section{Conclusions}

Increased WC is associated with persistent postoperative urinary symptoms after surgical treatment of BPE. Obese men have a higher risk of persistent storage LUTS after TURP or OP.

\section{Keywords}

benign prostatic enlargement, benign prostatic hyperplasia, lower urinary tract symptoms, metabolic syndrome, waist circumference

\section{Introduction}

Excess bodyweight has progressively increased since the 1980s, rapidly becoming a worldwide epidemic disorder with a remarkable socioeconomic impact, due to its association with increased morbidity and mortality [1].

Central obesity is also considered an early step in the development and progression of metabolic syndrome (MetS), a cluster of several medical conditions in addition to abdominal obesity, including diabetes, dyslipidaemia (high triglycerides and low high-density lipoprotein [HDL] cholesterol), autonomic hyperactivity and arterial hypertension [2]. In particular, visceral adipose tissue secretes various bioactive substances that can induce inflammatory responses and have proinflammatory effects. The progressive development of inflammation in men with MetS may explain the emerging link between MetS, benign prostatic enlargement (BPE) and LUTS [3-5].

In 1985, Glynn et al. [6] noted that in the Veterans Administration Normative Aging Study, only body mass 
index (BMI) was a significant predictor of a clinical diagnosis of BPH. Giovannucci et al. [7], 20 years ago, described the association between obesity (measured as waist circumference, WC) and BPH in a population of men followed for incidence of prostatectomy, suggesting that central obesity could worsen urinary symptoms and increase the risk of prostatic surgery. These data have recently been confirmed in a retrospective study showing that in patients with a larger WC there was an enhanced prevalence of MetS components, with the consequence of worsened voiding function [8].

The aim of the present multicentre prospective study was to evaluate the impact of the components of MetS on urinary outcomes after simple open prostatectomy (OP) or TURP for severe LUTS due to BPE.

\section{Patients and Methods}

Between January 2012 and September 2013, 378 consecutive men treated with simple OP or TURP for large BPE, were prospectively enrolled in two tertiary referral centres. In both high-volume referral centres, all patients included in this trial were managed by surgeons skilled in the diagnosis and treatment of BPE. The study did not require any deviation of the Good Clinical Practice and was conducted in accordance with the principles expressed in the Declaration of Helsinki. Inclusion criteria were: prostatectomy (TURP or OP) for moderate-to-severe LUTS due to BPE refractory to medical treatment, and ability to communicate and comply with study requirements. Exclusion criteria were: history of preceding prostate surgery, chronic medication for prostatitis and/or urinary infection or bladder stone, and known malignant disease including prostate cancer.

LUTS were measured by the IPSS and categorised as storage and voiding symptoms, immediately before surgery and at 612 months postoperatively. The total IPSS score was categorised into $0-7,8-19,20-35$; storage subscore into $0-5$, 6-9 and 10-15; voiding subscore into $0-6,7-13$ and $14-20$ as mild, moderate and severe, respectively. For all IPSS (total, storage and voiding) the percentage of recovery of urinary symptoms after the operation (total-, storage- and voiding- $\Delta$ IPSS) was calculated using the following method:

([Preoperative IPSS - Postoperative IPSS]/Preoperative IPSS), expressed as a percentage: the full recovery of lower urinary tract function was considered as $\Delta=100 \%$.

The PSA level and prostate volume (by ultrasound) were measured before hospitalisation. OP and TURP were performed as previously reported $[9,10]$.

\section{MetS Parameters}

MetS was defined according the USA National Cholesterol Education Program (NCEP)-Adult Treatment Panel III, which requires at least three of the following five components: central obesity (WC of $>102 \mathrm{~cm}$ ), elevated triglycerides $(\geq 1.7 \mathrm{mmol} / \mathrm{L}$ or $150 \mathrm{mg} / \mathrm{dL})$, elevated blood pressure ( $\mathrm{BP} \geq 130 / 85 \mathrm{mmHg}$ ), elevated fasting glucose $(\geq 6.1 \mathrm{mmol} / \mathrm{L}$ or $110 \mathrm{mg} / \mathrm{dL})$ and reduced HDL cholesterol $(<1.03 \mathrm{mmol} / \mathrm{L}$ or $40 \mathrm{mg} / \mathrm{dL})$ [11].

$\mathrm{WC}$ and $\mathrm{BP}$ were measured by trained personnel using a standardised protocol. In particular, WC was measured midway between the lowest rib and the iliac crest, to the nearest $0.1 \mathrm{~cm}$. Blood samples were drawn in the morning, after an overnight fast, for determination of blood glucose, total cholesterol, HDL cholesterol and triglycerides, 7-30 days preoperatively.

\section{Statistical Analyses}

The differences between men with MetS and without MetS were assessed by unpaired $t$-test (if normal) or with MannWhitney test (in the remaining cases). The correlations between all the recorded items and the improvement of urinary symptoms (total-, storage- and voiding- $\Delta$ IPSS) were calculated by a Spearman correlation analysis; significant data were included in a multivariate logistic regression analysis. Moreover, we included significant data in a binary logistic model to evaluate the odds ratio (OR) based on full recovery of lower urinary tract function ( $\triangle$ IPSS $=100 \%$ ) vs partial recovery ( $\triangle$ IPSS $<100 \%)$. All the analyses were done with SPSS statistics 17.0 version for windows XP and a $P<0.05$ was considered to indicate statistical significance.

\section{Results}

In all, 378 unselected consecutive men were recruited in two tertiary referral centres for surgical treatment of BPE. Overall, 191 patients (50.5\%) presented moderate LUTS, while 187 (49.5\%) had severe LUTS. According to the IPSS subscores, $53(14.3 \%), 211(55.7 \%)$ and $114(30 \%)$ patients presented with mild, moderate or severe voiding LUTS, respectively. In addition, 80 (21.2\%), 149 (39.4\%) and 149 (39.4\%) presented with mild, moderate or severe storage LUTS, respectively. Preoperative patient's characteristics, stratified according to MetS diagnosis, are reported in Table 1. As expected, there was a significant difference between the population according to weight and BMI (both $P<0.001$ ). Patients with MetS showed milder preoperative voiding symptoms and worse postoperative storage symptoms as compared with those without MetS (Table 1). In addition, an increasing number of MetS components resulted in increased postoperative storage IPSS scores (age-adjusted Wald 1.090, $P=0.009$; Fig. 1).

Overall, 220 patients (58.2\%) reported a complete recovery of their lower urinary tract function (total- $\triangle$ IPSS $100 \%$ ), while the remaining $158(41.8 \%)$ had an incomplete recovery; in particular, 222 men (58.7\%) reported a complete recovery of 
Table 1 Descriptive statistics of population of men included in the study, stratified according to their MetS profile.

\begin{tabular}{lccc}
\hline Patients $(\boldsymbol{n}=\mathbf{3 7 8})$ & $\begin{array}{c}\text { With MetS } \\
(\boldsymbol{n}=\mathbf{1 4 0}) \\
\text { Mean (sD) }\end{array}$ & $\begin{array}{c}\text { Without MetS } \\
(\boldsymbol{n}=\mathbf{2 3 8}) \\
\text { Mean (SD) }\end{array}$ & $\mathbf{P}$ \\
\hline Demographic & & & \\
$\quad$ Age, years & $69.7(7.4)$ & $68.5(8.8)$ & 0.187 \\
$\quad$ Weight, kg & $81.5(12.0)$ & $77.3(8.5)$ & 0.000 \\
Height, m & $1.72(0.1)$ & $1.73(0.1)$ & 0.136 \\
$\quad$ BMI, kg/m & $27.4(3.5)$ & $25.7(2.3)$ & $<0.001$ \\
Prostate features & & & \\
$\quad$ Prostate volume, mL & $79.1(42.3)$ & $88.6(58.2)$ & 0.110 \\
$\quad$ PSA level, ng/mL & $2.6(3.0)$ & $1.9(1.9)$ & 0.143 \\
Preoperative uroflowmetry & & & \\
$\quad$ Qmax, mL/s & $8.9(3.5)$ & $8.8(3.6)$ & 0.905 \\
$\quad$ Qave, mL/s & $5.5(2.4)$ & $5.2(2.2)$ & 0.438 \\
$\quad$ Voided volume, mL & $248(46.5)$ & $257(58.3)$ & 0.398 \\
Preoperative IPSS & & & \\
$\quad$ Total & $20.0(5.7)$ & $20.5(4.8)$ & 0.454 \\
$\quad$ Voiding & $11.1(4.2)$ & $12.3(4.1)$ & 0.024 \\
$\quad$ Storage & $8.9(3.7)$ & $8.7(3.2)$ & 0.603 \\
Postoperative IPSS & & & \\
$\quad$ Total & $2.2(3.8)$ & $1.5(3.3)$ & 0.077 \\
$\quad$ Voiding & $0.5(1.7)$ & $0.6(1.1)$ & 0.413 \\
$\quad$ Storage & $2.3(3.6)$ & $1.3(2.5)$ & 0.015 \\
\hline
\end{tabular}

$Q_{a v e,}$ average urinary flow rate; $Q_{\max }$ maximum urinary flow rate.

Fig. 1 The mean and $95 \% \mathrm{Cl}$ of the mean of postoperative IPSS storage score, stratified according to the number of MetS parameters (ageadjusted Wald 1.090, $P=0.009$ ).

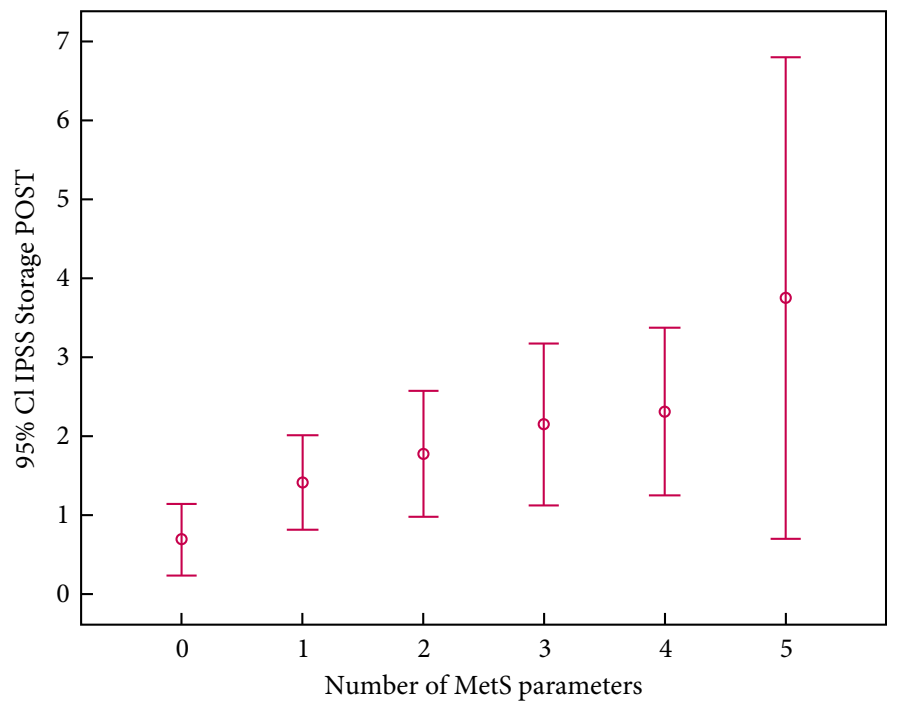

their storage symptoms and 293 (77.5\%) of their voiding symptoms. According to the postoperative IPSS subscores, 355 (93.9\%), 20 (5.3\%) and three (0.8\%) patients had mild, moderate or severe LUTS, respectively. Categorising for voiding and storage IPSS, 372 (98.4\%) and six (1.6\%) patients, had mild or moderate voiding LUTS, while 350 (92.7\%), 13 (3.5\%) and 15 (3.8\%) patients presented mild, moderate or severe storage LUTS, respectively. Moreover,
Table 2 Multivariate regression analysis adjusted for age, PSA level, volume, smoking history, to evaluate the impact of number of MetS parameters and each component on the improvement of total IPSS (total$\triangle$ IPSS).

\begin{tabular}{lcccc}
\hline IPSS total & $\begin{array}{l}\text { B (non-standardised } \\
\text { coefficient) }\end{array}$ & $\begin{array}{l}\text { Lower } \\
\text { limit }\end{array}$ & $\begin{array}{l}\text { Upper } \\
\text { limit }\end{array}$ & $P$ \\
\hline MetS (0-5) & -0.047 & -0.095 & 0.001 & 0.052 \\
WC & -0.008 & -0.013 & -0.003 & 0.003 \\
Diastolic BP & -0.013 & -0.022 & -0.005 & 0.003 \\
Systolic BP & -0.005 & -0.010 & 0.000 & 0.057 \\
Glycaemia & 0.000 & -0.002 & 0.002 & 0.955 \\
Triglyceride & 0.000 & -0.002 & 0.001 & 0.708 \\
HDL-cholesterol & -0.002 & -0.008 & 0.004 & 0.547 \\
\hline
\end{tabular}

Table 3 Multivariate regression analysis adjusted for age, PSA, volume, smoking history, to evaluate the impact of number of MetS parameters and each component on the improvement of storage IPSS (storage- $\Delta$ IPSS).

\begin{tabular}{lcccc}
\hline IPSS storage & $\begin{array}{l}\text { B (non-standardised } \\
\text { coefficient) }\end{array}$ & $\begin{array}{l}\text { Lower } \\
\text { limit }\end{array}$ & $\begin{array}{l}\text { Upper } \\
\text { limit }\end{array}$ & $\boldsymbol{P}$ \\
\hline MetS (0-5) & -0.130 & -0.240 & -0.020 & 0.021 \\
WC & -0.021 & -0.033 & -0.009 & 0.001 \\
Diastolic BP & -0.027 & -0.046 & -0.008 & 0.006 \\
Systolic BP & -0.010 & -0.022 & 0.001 & 0.074 \\
Glycaemia & 0.001 & -0.005 & 0.006 & 0.742 \\
Triglyceride & 0.000 & -0.004 & 0.003 & 0.895 \\
HDL-cholesterol & -0.005 & -0.018 & 0.009 & 0.518 \\
\hline
\end{tabular}

Table 4 Multivariate regression analysis adjusted for age, PSA, volume, smoking history, to evaluate the impact of number of MetS parameters and each component on the improvement of voiding IPSS (voiding- $\Delta$ IPSS).

\begin{tabular}{lcccc}
\hline IPSS voiding & $\begin{array}{l}\text { B (non-standardised } \\
\text { coefficient) }\end{array}$ & $\begin{array}{l}\text { Lower } \\
\text { limit }\end{array}$ & $\begin{array}{l}\text { Upper } \\
\text { limit }\end{array}$ & $\boldsymbol{P}$ \\
\hline MetS (0-5) & 0.001 & -0.03 & 0.031 & 0.973 \\
WC & -0.003 & -0.006 & 0.001 & 0.112 \\
Diastolic BP & -0.009 & -0.014 & -0.004 & 0.001 \\
Systolic BP & -0.003 & -0.006 & 0.000 & 0.077 \\
Glycaemia & 0.001 & -0.001 & 0.002 & 0.336 \\
Triglyceride & 0.000 & -0.001 & 0.001 & 0.962 \\
HDL-cholesterol & -0.001 & -0.005 & 0.003 & 0.700 \\
\hline
\end{tabular}

patients with MetS reported a lower recovery in total and storage IPSS as compared with those without MetS (63.2 vs $81.7, P=0.015$ and 19.6 vs $63.6, P=0.013$, respectively).

Improvements in the total and storage IPSS were related to WC and diastolic BP (Tables 2 and 3), while improvement in voiding IPSS was only related to diastolic BP (Table 4), after adjustment for age, smoking history, PSA level and prostate volume. These data were confirmed after further adjustment for the numbers of MetS factors (data not shown).

In the binary logistic model, adjusted for age, smoking history, PSA level, prostate volume, presence of MetS and surgical procedure, men with a WC of $>102 \mathrm{~cm}$ had a higher risk of an incomplete recovery of lower urinary tract function, 
Fig. 2 OR based on full recovery of lower urinary tract function (total- $\Delta$ IPSS 100\%) vs partial recovery (total- $\Delta$ IPSS $<100 \%$ ), as derived from a logistic regression model adjusted for: Age, PSA level, prostate volume, smoking history, surgical procedure (TURP or OP), presence of MetS. LL, lower limit; UL, upper limit.

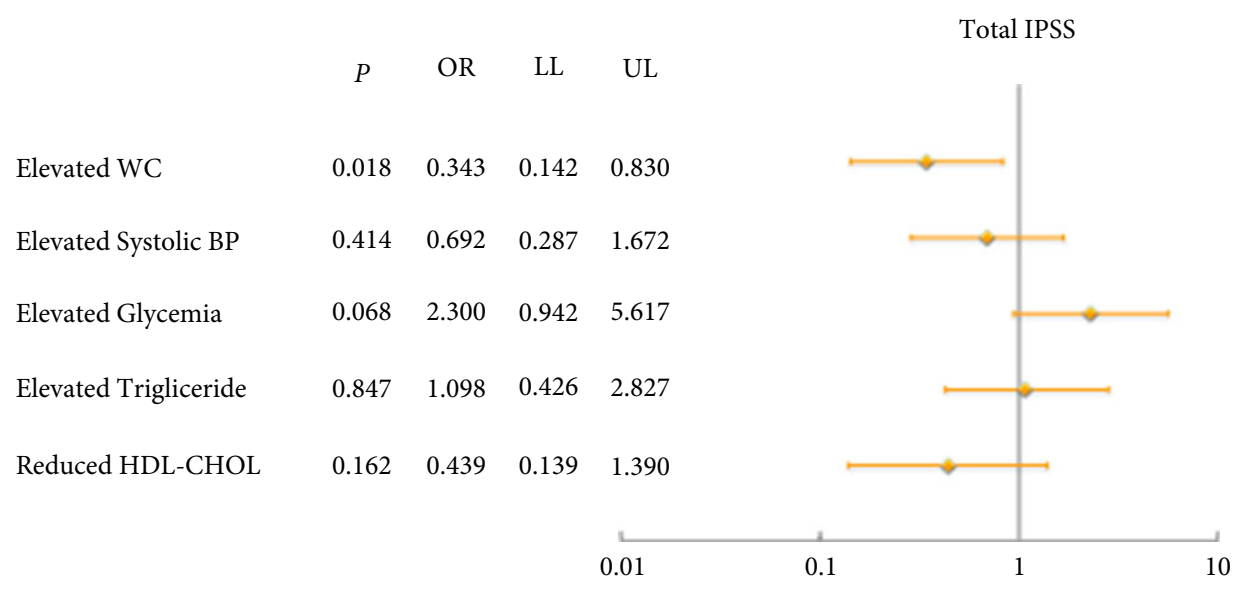

Fig. 3 OR based on full recovery of lower urinary tract function (storage- $\Delta$ IPSS 100\%) vs partial recovery (storage- $\Delta$ IPSS $<100 \%$ ), as derived from a logistic regression mode adjusted for: Age, PSA level, prostate volume, smoking history, surgical procedure (TURP or OP), presence of MetS. LL, lower limit; UL, upper limit.

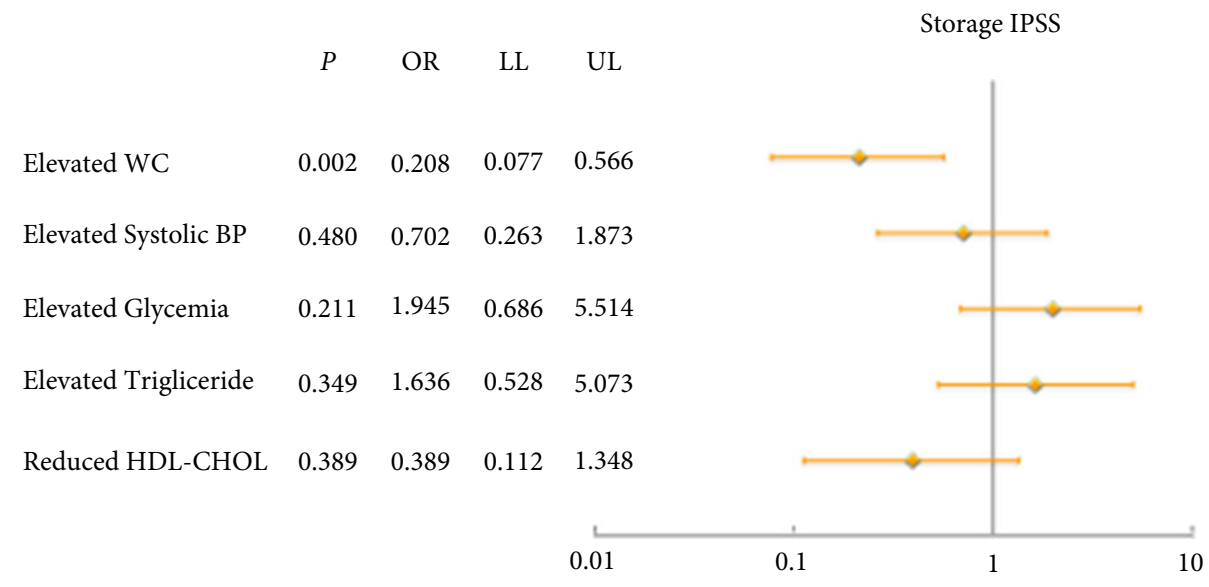

measured with total IPSS (OR 0.343, $P=0.018$; Fig. 2) and storage IPSS (OR 0.208, $P=0.002$; Fig. 3), as compared with those with a WC of $<102 \mathrm{~cm}$. No MetS parameter could be identified as a determining factor for full recovery of voiding function (Fig. 4).

\section{Discussion}

Central obesity and MetS are becoming critical topics in urology due to their global epidemic diffusion and strong relationship with several urological diseases [12]. In the 'Multicenter Italian Report on Radical prostatectomy: Outcome and Research' (MIRROR) study, based on data from men treated with radical prostatectomy for prostate cancer in 25 urological departments across the whole Italian territory, we have previously shown a significant positive correlation between WC and post-prostatectomy incontinence severity; the risk of needing at least two pads/day is 2.5-times greater in men with a WC of $\geq 102 \mathrm{~cm}$ than those with a WC of $<102 \mathrm{~cm} \mathrm{[13].}$

While following 5667 men in the Prostate Cancer Prevention Trial placebo arm, investigators noted the predictors for progression were age, baseline LUTS severity, an elevated hip-waist ratio, as well as ethnic background. Specifically they noted the risk of LUTS worsening (defined as 2 points worse on the IPSS $)$ increased by $4 \%(P<0.001)$ with each additional year of age. Additional risks for worsening LUTS were $41 \%$ higher for Black $(P<0.03)$ and Hispanic men $(P<$ 0.06) compared with White men, and for severe LUTS these increases were 68\% $(P<0.01)$ and 59\% $(P<0.03)$, respectively. Each 0.05 increase in waist-hip ratio (a measure 
Fig. 4 OR based on full recovery of lower urinary tract function (voiding- $\Delta$ IPSS 100\%) vs partial recovery (voiding- $\Delta$ IPSS $<100 \%$ ), as derived from a logistic regression mode adjusted for: Age, PSA level, prostate volume, smoking history, surgical procedure (TURP or OP), presence of MetS. LL, lower limit; UL, upper limit.

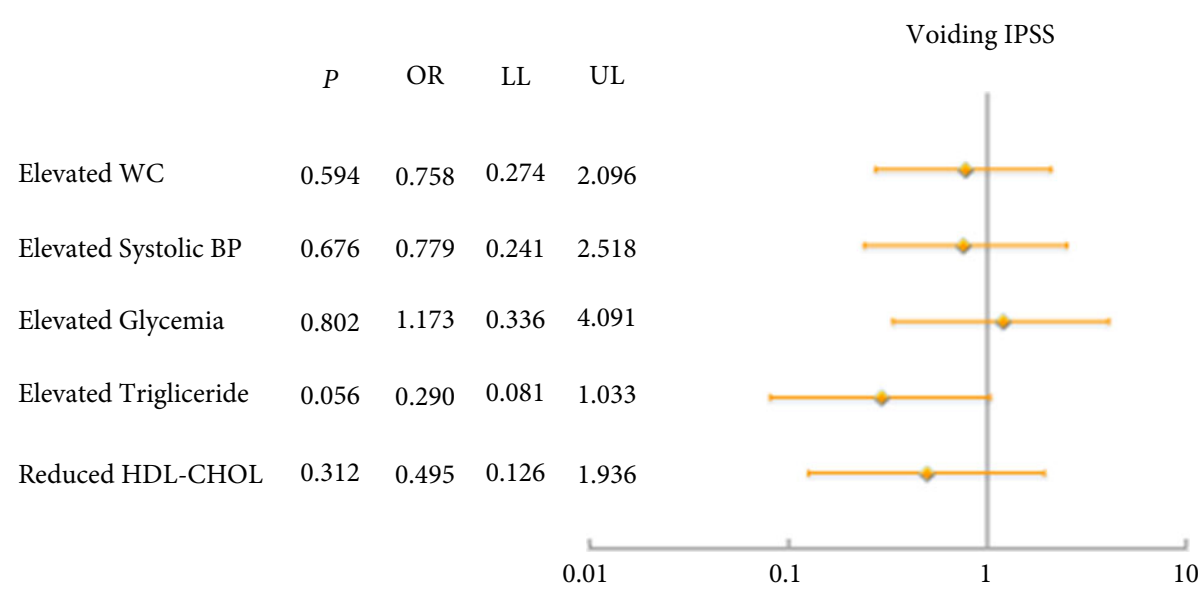

of abdominal obesity) was associated with a $10 \%$ increased risk of overall LUTS progression $(P<0.003)$ and severe LUTS $(P<0.02)$. They noted that weight loss might be helpful for the treatment and prevention of LUTS [14]. A cohort of men undergoing TURP were noted to have a higher waist-hip ratio than controls; relative to men in the lowest waist-hip ratio quartile $(<0.856)$ those in the highest quartile $(>0.923)$ were at 2.4-fold risk of BPH (OR 2.42, 95\% CI 1.34 4.37, test for trend $P=0.01$ ) [15].

Recently, several studies have focused on the impact of MetS parameters on LUTS due to BPE [15-17]. We have recently shown that MetS can lead to an increase in prostate volume. In particular, our meta-regression analysis showed that the overall prostate volume and the transitional zone increment were significantly greater in older and obese men, and in those with low serum HDL cholesterol levels [18].

Moreover, as reported by Lee et al. [8], an increased WC was associated with worsened voiding function. In particular, the multivariate logistic regression analysis of urological parameters with WC showed that as WC increased from $<90$ to $90-99$ to $\geq 100 \mathrm{~cm}$, there was a greater likelihood for prostate volume growth (OR 1.39, $P=0.01$ ).

In the present study, we report a unique finding, where the worse outcomes after surgery for BPH/LUTS were in men with increased WC. Men with a WC of $\geq 102 \mathrm{~cm}$ had threefold risk of an incomplete recovery of LUTS and a five-fold risk of having persistent storage symptoms after surgery. In our unselected consecutive cross-sectional population, men with MetS presented similar baseline IPSS storage scores (8.9 vs 8.7 ) as compared with men without MetS: nevertheless, postoperatively they presented significantly worse IPSS storage scores than those without MetS (2.3 vs 1.3). A comparable correlation between the number of components of MetS and the severity of urinary symptoms has been recently reported in a prospective cross-sectional study of men aged 50-59 years who had participated in a hospital health examination [19]. The authors reported that the number of men with LUTS (IPSS $>7$ ), enlarged prostate volume (total prostate volume $\geq 30 \mathrm{~mL}$ ) and/or reduced maximum urinary flow rate $\left(Q_{\max }<15 \mathrm{~mL} / \mathrm{s}\right)$ significantly increased with an increasing number of metabolic abnormalities. These results corroborated our preclinical data, suggesting that MetS could have a detrimental effect on prostate health, boosting inflammation, and finally inducing the concomitant enlargement of the prostatic gland and the decline of storage LUTS [20].

We recently suggested that hormonal, inflammatory and metabolic derangements might have a permissive or causative role in determining BPH. In particular, MetS could induce or maintain an inflammatory state within the prostate that could even be exacerbated by a relative hyperoestrogenism [21] or by androgen deficiency [22,23], medical conditions often associated to MetS and in particular to increased WC [24,25].

In the present study, diastolic BP emerges as a negative predictive factor for both voiding and storage urinary symptoms, while WC seems to have a negative impact exclusively on storage symptoms (Tables 2, 3 and 4). Interestingly, these data are consistent to those reported in a recently published observational study on police officers in their 50s with moderate-to-severe LUTS (IPSS >7) [26]. Their logistic regression analysis showed that only WC and hypertension among the MetS components were determinants of progression of BPH (OR 1.034, $P=0.021$ and OR 1.511, $P$ $=0.009$, respectively). Moreover, the clinical impact of BP on persistent storage LUTS after TURP has been recently highlighted by a prospective study on patients treated with TURP for BOO due to BPH, based on the use of pulsed wave colour Doppler ultrasonography. The author showed that 
persistent detrusor overactivity, 1 year after TURP, was associated with increased vascular resistance of the bladder vessels with subsequent reduced perfusion and hypoxia [27].

We recently showed in an experimental animal model of high fat diet-induced MetS, that MetS was associated with severe vascular damage in the whole of the LUT. In particular, we observed severe inflammation, running in parallel with hypoxia and tissue remodelling, both in prostatic and bladder tissue $[22,28]$.

The binary logistic model showed that in cases of severe LUTS due to large BPE, the full recovery of LUTS and especially that of storage symptoms, can be more easily achieved in men with a WC of $<102 \mathrm{~cm}$ compared with those with WC of $\geq 102 \mathrm{~cm}$. Moreover, central obesity, as with other MetS parameters, seems to have no detrimental effect on the recovery of voiding activity, as measured by the IPSS. Thus surgical removal of central adenomatous tissue allows one to largely reverse flow-related symptoms, regardless of MetS features.

Our present prospective study has several limitations. We included men treated exclusively in tertiary referral centres for BPE surgery; this population of men with large prostates $(\geq 80 \mathrm{~mL})$, and a pronounced reduction in urinary flow $\left(Q_{\max }\right.$ $<9 \mathrm{~mL} / \mathrm{s}$ ) may be very different to that of the general community. Furthermore, we evaluated the population exclusively with the IPSS. The use of other validated questionnaires, such as the Short Form-12 to evaluate overall health (including physical and mental health), might have provided additional insight. Moreover, data from preoperative and postoperative pressure/flow studies were not available. Finally, we did not adjust our present data for additional parameters, such as physical activity and comorbidities, which can influence the development of LUTS or alter clinical outcomes after any surgical procedure.

In conclusion, our present study demonstrated that increased WC is associated with worse urinary symptoms recovery after surgical treatment of BPE. In particular, obese men have a higher risk of persistent storage LUTS after TURP or OP. Further prospective studies are needed to confirm our present data.

\section{Conflicts of Interest}

No sources of funding were used to assist in the preparation of this article. The Authors have no conflict of interest for this study.

\section{References}

1 Finucane MM, Stevens GA, Cowan MJ et al. Global Burden of Metabolic Risk Factors of Chronic Diseases Collaborating Group (Body Mass Index): National, regional, and global trends in body-mass index since 1980: systematic analysis of health examination surveys and epidemiological studies with 960 country-years and 9.1 million participants. Lancet 2011; 377: 557-67

2 Eckel RH, Alberti KG, Grundy SM, Zimmet PZ. The metabolic syndrome. Lancet 2010; 375: 181-3

3 Gacci M, Vignozzi L, Sebastianelli A et al. Metabolic syndrome and lower urinary tract symptoms: the role of inflammation. Prostate Cancer Prostatic Dis 2013; 16: 101-6

4 Bostanci Y, Kazzazi A, Momtahen S, Laze J, Djavan B. Correlation between benign prostatic hyperplasia and inflammation. Curr Opin Urol 2013; 23: 5-10

5 Gandaglia G, Briganti A, Gontero P et al. The role of chronic prostatic inflammation in the pathogenesis and progression of benign prostatic hyperplasia (BPH). BJU Int 2013; 112: 432-41

6 Glynn RJ, Campion EW, Bouchard GR, Silbert JE. The development of benign prostatic hyperplasia among volunteers in the Normative Aging Study. Am J Epidemiol 1985; 121: 78-90

7 Giovannucci E, Rimm EB, Chute CG et al. Obesity and benign prostatic hyperplasia. Am J Epidemiol 1994; 140: 989-1002

8 Lee RK, Chung D, Chughtai B, Te AE, Kaplan SA. Central obesity as measured by waist circumference is predictive of severity of lower urinary tract symptoms. BJU Int 2012; 110: 540-5

9 Gacci M, Bartoletti R, Figlioli S et al. Urinary symptoms, quality of life and sexual function in patients with benign prostatic hypertrophy before and after prostatectomy: a prospective study. BJU Int 2003; 91: 196-200

10 Tubaro A, Carter S, Hind A, Vicentini C, Miano L. A prospective study of the safety and efficacy of suprapubictransvesical prostatectomy in patients with benign prostatic hyperplasia. J Urol 2001; 166: 172-6

11 Expert Panel on Detection. Evaluation, and treatment of high blood cholesterol in adults. Executive Summary of the Third Report of the National Cholesterol Education Program (NCEP) Expert Panel on Detection, Evaluation, and Treatment of High Blood Cholesterol In Adults (Adult Treatment Panel III). JAMA 2001; 285: 2486-97

12 De Nunzio C, Aronson W, Freedland SJ, Giovannucci E, Parsons JK. The correlation between metabolic syndrome and prostatic diseases. Eur Urol 2012; 61: 560-70

13 Gacci M, Sebastianelli A, Salvi M et al. Role of abdominal obesity for functional outcomes and complications in men treated with radical prostatectomy for prostate cancer: results of the Multicenter Italian Report on Radical Prostatectomy (MIRROR) study. Scand J Urol 2014; 48: 13845

14 Kristal AR, Arnold KB, Schenk JM et al. Race/ethnicity, obesity, health related behaviors and the risk of symptomatic benign prostatic hyperplasia: results from the prostate cancer prevention trial. J Urol 2007; 177: 1395-400

15 Dahle SE, Chokkalingam AP, Gao YT et al. Body size and serum levels of insulin and leptin in relation to the risk of benign prostatic hyperplasia. J Urol 2002; 168: 599-604

16 Hammarsten J, Högstedt B, Holthuis N, Mellström D. Components of the metabolic syndrome-risk factors for the development of benign prostatic hyperplasia. Prostate Cancer Prostatic Dis 1998; 1: 157-62

17 Ozden C, Ozdal OL, Urgancioglu G, Han O, Seckin S, Memis A. The correlation between metabolic syndrome and prostatic growth in patients with benign prostatic hyperplasia. Eur Urol 2007; 51: 199-203

18 Gacci M, Corona G, Vignozzi L et al. Metabolic syndrome and benign prostatic enlargement: a systematic review and meta-analysis. BJU Int 2015; 115: 24-31

19 Park YW, Kim SB, Kwon H et al. The relationship between lower urinary tract symptoms/benign prostatic hyperplasia and the number of components of metabolic syndrome. Urology 2013; 82: 674-9 
20 Vignozzi L, Gacci M, Cellai I et al. Fat boosts, while androgen receptor activation counteracts. BPH-associated prostate inflammation. Prostate 2013; 73: 789-800

21 Comeglio P, Morelli A, Cellai I et al. Opposite effects of tamoxifen on metabolic syndrome-induced bladder and prostate alterations: a role for GPR30/GPER? Prostate 2014; 74: 10-28

22 Vignozzi L, Morelli A, Sarchielli E et al. Testosterone protects from metabolic syndrome-associated prostate inflammation: an experimental study in rabbit. J Endocrinol 2012; 212: 71-84

23 Vignozzi L, Cellai I, Santi R et al. Antiinflammatory effect of androgen receptor activation in human benign prostatic hyperplasia cells. $J$ Endocrinol 2012; 214: 31-43

24 Vignozzi L, Rastrelli G, Corona G, Gacci M, Forti G, Maggi M. Benign prostatic hyperplasia: a new metabolic disease? J Endocrinol Invest 2014; 37: $313-22$

25 Corona G, Vignozzi L, Rastrelli G, Lotti F, Cipriani S, Maggi M. Benign prostatic hyperplasia: a new metabolic disease of the aging male and its correlation with sexual dysfunctions. Int J Endocrinol 2014; 2014: 329456

26 Kwon H, Kang HC, Lee JH. Relationship between predictors of the risk of clinical progression of benign prostatic hyperplasia and metabolic syndrome in men with moderate to severe lower urinary tract symptoms. Urology 2013; 81: 1325-9

27 Mitterberger M, Pallwein L, Gradl J et al. Persistent detrusor overactivity after transurethral resection of the prostate is associated with reduced perfusion of the urinary bladder. BJU Int 2007; 99: 831-5

28 Morelli A, Comeglio P, Filippi S et al. Testosterone and farnesoid X receptor agonist INT-747 counteract high fat diet-induced bladder alterations in a rabbit model of metabolic syndrome. J Steroid Biochem Mol Biol 2012; 132: 80-92

Correspondence: Mauro Gacci, Department of Urology, University of Florence, Careggi Hospital, Viale Pieraccini 18, 50139 Florence, Italy.

e-mail: maurogacci@yahoo.it

Abbreviations: BMI, body mass index; BP, blood pressure; $\mathrm{BPE}$, benign prostatic enlargement; HDL, high-density lipoprotein; MetS, metabolic syndrome; OR, odds ratio; $Q_{\max }$, maximum urinary flow rate; WC, waist circumference. 